\title{
Cavity Engineering for Superconductors
}

\section{Enclosing a cuprate superconductor in a passive THz-resonant cavity could provide a new route to modifying a superconductor's properties.}

\author{
By Marric Stephens
}

$W^{2}$

esearchers working on superconductivity would like to induce the phenomenon at practical temperatures.

In this effort, they tweak the compositions of known superconductors, develop new material classes, and subject candidate compounds to enormous pressures. Jonathan Curtis at Harvard University and colleagues now propose an alternative approach [1]. They predict a way to manipulate, using THz-resonant cavities, the quasiparticles that promote superconductivity in certain superconductors. While they don't yet know if the idea will work, they hope it could raise a superconductor's critical temperature.

The idea of modifying a superconductor's properties using an electromagnetic cavity has been proposed before. The principle relies on the fact that in a conventional superconductor, the binding of electrons into Cooper pairs is mediated by phonons, which can be manipulated by coupling them to a cavity mode. But conventional superconductors have such low critical temperatures that no realistically attainable increase would make them practical. Curtis and colleagues

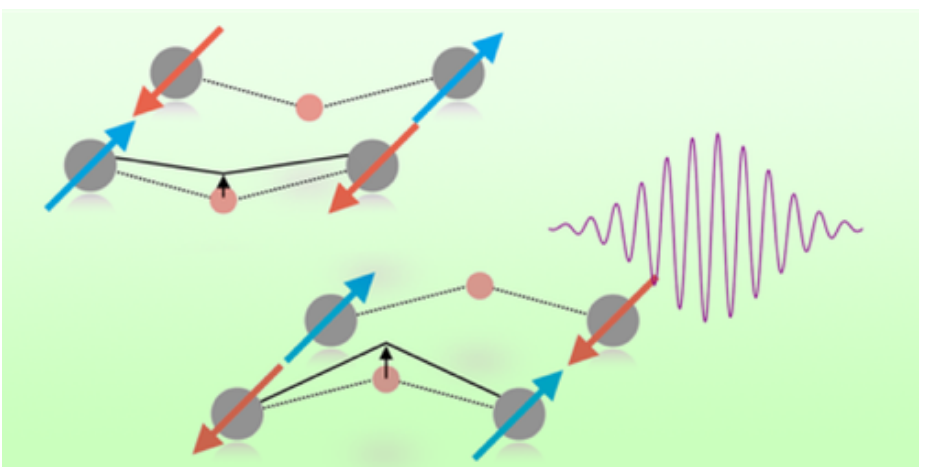

Credit: J. B. Curtis et al. [1] instead investigate an unconventional superconductor-the bilayer cuprate $\mathrm{YBa}_{2} \mathrm{Cu}_{3} \mathrm{O}_{6}$-which starts with the more promising critical temperature of nearly $100 \mathrm{~K}$.

In a cuprate superconductor, Cooper-pair formation is thought to be mediated by magnons rather than phonons. The team considers two mechanisms by which cavity-phonon coupling could affect these magnons. The strongest effect (leading, they hope, to the greatest temperature change) is predicted for $\mathrm{YBa}_{2} \mathrm{Cu}_{3} \mathrm{O}_{6}$ and other bilayer cuprates in which the cavity-phonon-magnon interaction arises from lattice distortions. A weaker effect is predicted for all cuprates-including monolayer cuprates-in which the phonons excite magnons via a spin-orbit interaction. Curtis and colleagues don't know whether critical temperatures really can be changed in this way. But, they say, testing their prediction experimentally would be the first step toward obtaining such control.

Marric Stephens is a Corresponding Editor for Physics based in Bristol, UK.

\section{REFERENCES}

1. J. B. Curtis et al., "Cavity magnon-polaritons in cuprate parent compounds," Phys. Rev. Research 4, 013101 (2022). 